\title{
Lifting Wavelet Transform for Super Resolution Image Reconstruction using MATLAB
}

\author{
B. Janardhana Rao ${ }^{1}$ and O. Venkata Krishna ${ }^{2}$ \\ ${ }^{1}$ CVR College of Engineering/ECE, Hyderabad, India \\ Email: janardhan.bitra@gmail.com \\ ${ }^{2}$ CVR College of Engineering/EIE, Hyderabad, India \\ Email: venkatakrishna.odugu@gmail.com
}

\begin{abstract}
The aim of this paper is to develop a high resolution image from a sequence of low resolution compressed images. An image with improved resolution is desired in almost all of the applications to enhance qualitative features and is reported to be achieved by Super Resolution Image Reconstruction (SRIR). Some low resolution images of same scene which are usually rotated, translated and blurred are taken to form a super resolution image.

The image registration operation orients translated, scaled and rotated images in similar way to that of source image. Lifting Wavelet Transform (LWT) with Daubechies4 coefficients is applied to color components of each image due to its less memory allocation compared to other techniques. Further Set Portioning in Hierarchical Trees (SPIHT) algorithm is applied for image compression as it possess lossless compression, fast encoding/decoding, adaptive nature. The three low resolution images are fused by spatial image fusion method. The noise component is removed by dual tree Discrete Wavelet Transform (DWT) and blur is removed by blind de-convolution or iterative blind de-convolution. Finally, the samples are interpolated to twice the number of original samples to obtain a super resolution image. The structural similarity for each intermediate image compared to source image is estimated via objective analysis and high structural similarity is observed for image constructed by the proposed method.
\end{abstract}

Index Terms-Wavelets, Lifting Scheme, Super Resolution, Daubechies, Image Registration, SPIHT

\section{INTRODUCTION}

Super resolution is nothing but improving the resolution of an image. High resolution images are widely used in most of the image processing applications such as remote surveillance video enhancement, industrial inspection, medical imaging, robot vision and remote sensing. Super Resolution is the technique of producing a higher spatial resolution image from one or more under sampled low resolution images, low resolution refers to the less pixel density in an image which gives very few details. The purpose behind the reconstruction of a single high resolution image from several low resolution images is that the images are degraded and mis-registered such as blur and noise, these images are sub pixel shifted due to sub-sampling which results in an aliased low resolution image. From these sub pixel shifted images, a variety of information can be collected from each low resolution image. A simple version of SRIR Model is shown in figure 1 [1]

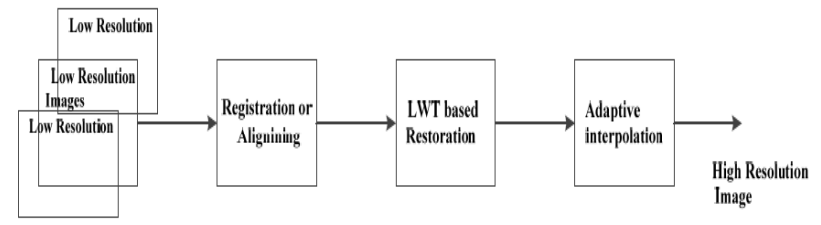

Figure 1: Super Resolution Image Reconstruction Model.

Super Resolution is an emerging technology in signal processing to get a High Resolution (HR) image. The main aim of Super Resolution (SR) is to enhance the spatial resolution of multiple lower resolution images. The super resolution technique is an efficient lossy and low cost technology. In this paper we are using Wavelet Transform (WT) technique to get an HR image from Low Resolution (LR) images by involving image registration, blurring, decimation, re-registration, de-blurring, denoising and interpolation operation.

The major challenges for super resolution are image registration, computation efficiency, robustness aspects, and speed issue (fast algorithm implementation).

\section{IMAGE REGISTRATION}

Image registration is a process of overlaying two or more images of the same scene taken at different times, from different viewpoints and by different sensors. Source image is taken as the reference and compared with the input images. The main goal is to make the input image appear as the source image by applying spatial transformations which help in mapping the locations in one image to the new locations in the other. Image registration mainly depends on the properties of the capturing device such as sampling rate, resolution of the sensor, the imperfection of the lens that adds blur and the noise of the device used for image acquisition. An accurate registration of two low resolution images becomes more difficult because If the resolution of an image goes down, the two dimensional structure of an image also goes down. Here the registration technique used is based on Fast Fourier Transform proposed by Fourier Mellin, DeCastro and Morandi. Transformation used is rotation, translation and shift estimation and the process of the Image Registration is shown in Figure 2 [2]. 


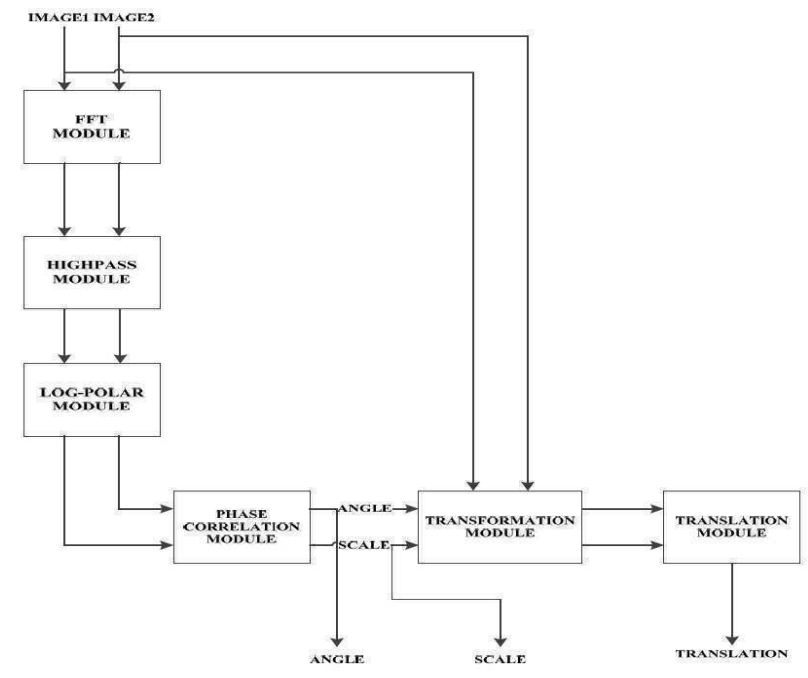

Figure 2: Image Registration Algorithm.

\section{LIFTING WAVELET TRANSFORM}

Wavelet is a function of finite length and is oscillatory. The dilations and translations of a source wavelet are used to operate the spatial or frequency analysis of the input data in wavelet transform. Wavelets are widely used in major applications, since the image quality can be improved by the addition of detailed information. This makes wavelets more attractive for progressive transmission of images. Wavelets build the complex filters to generate the data which can improve the quality of the specified region of the image. Wavelet techniques found applications in noise reduction, signal variance estimation, frequency analysis and also for data compression. One of the features of wavelet techniques is the perfect reconstruction. This means extraction of original data set from the result of inverse wavelet transform. The wavelet transforms can be used in image processing due to their capability of reducing the size (storage) of the image without affecting the image resolution which can save computation time [3].

Lifting is a transform that makes use of means and differences. The mean values have a control over the entire structure of the signal and termed as update and in differences, if the difference between two samples is almost same or very small, then it can be concluded that the first sample is the prediction of the second one. The wavelet lifting scheme is a method that separates the wavelet transform into a set of steps.

The forward wavelet transform is considered in forward lifting wavelet transform, the data under process is divided into an odd and an even element.

The simple form of a forward lifting wavelet transform is shown in figure 3 [5]. The process is initiated with a finite sequence $S_{j}$ of length $2^{\mathrm{j}}$. It is transformed into two sequences, each of length $2^{j-1}$ and denoted $S_{j-1}$ and $d_{j-1}$, respectively.

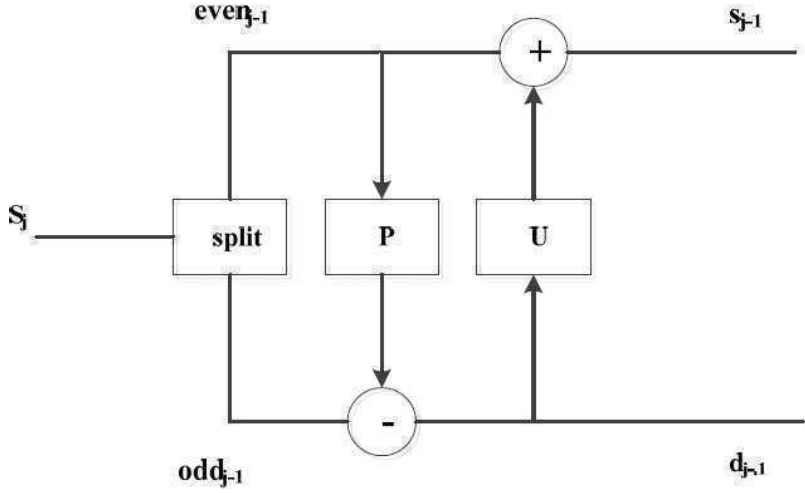

Figure 3: Three step lifting scheme.

Note that the minus represents 'the signal from the left minus the signal from the top'

The following are the three steps of lifting scheme

Split The split step sort the entries into the even and the odd entries. The functionality of the algorithm is made clear in this split operation. In effective implementation the entries are not moved or separated.

Predict A correlation between the sample and its nearest neighbors is predicted when the signal containing some structure. Given at the sample number $2 \mathrm{n}$, we predict that the value at sample $2 n+1$ is the same. When the value at $2 n+1$ is replaced with the correction to the prediction, which is the difference.

$$
d j-1[n]=s j[2 n+1]-s j[2 n]
$$

In general, the idea is to have a prediction procedure $P$ and then compute

$$
d j-1=\text { odd } j-1-p(\text { evenj }-1)
$$

Thus in the d signal each entry is one odd sample minus some prediction based on a number of even samples.

Update For a given even entry, a prediction is made that the next odd entry has the small value and the difference is stored. Then an update of the even entry is done to show our knowledge of the signal.

$$
s j-1[n]=s j[2 n]+d j-1[n] / 2
$$

In general, a suitable updating procedure is chosen and then used in computing

$$
s j-1=\text { even } j-1+U(d j-1)
$$

The Predict step finds the wavelet functions in the wavelet transform. This is a High pass filter. The Update step finds the scaling function resulting to a smoother version of the data. This is a Low pass filter.

A combination of number of lifting steps yields the DWT. The computed differences $D_{j-1}$ are left behind and the average sequence $\mathrm{s}_{\mathrm{j}-1}$ of the previous lifting step is used as input for the preceding lifting step. The two step discrete wavelet transform is shown in the figure 4 [5] 


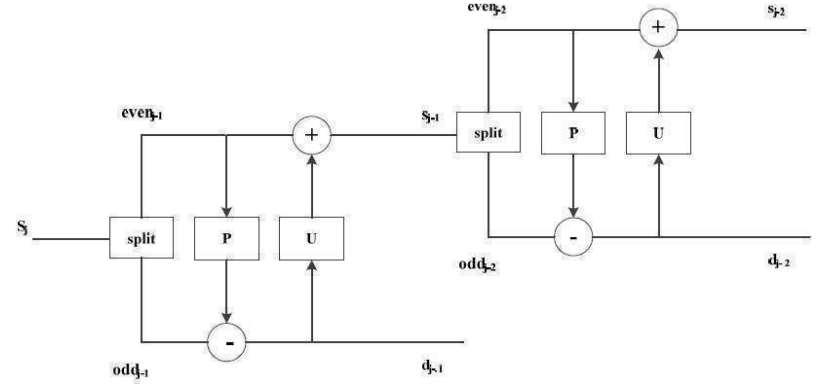

Figure 4: Two-steps Discrete Wavelet Transform.

The Inverse Lifting Scheme is a mirror of Forward Lifting Scheme and is checked by reversing the arrows and changing the signs. The direct form is

$$
\begin{aligned}
& d j-1=\text { odd }-1-P(\text { evenj }-1) \\
& s j-1=\text { evenj }-1+U(\text { dj }-1)
\end{aligned}
$$

is inverted by the steps

$$
\begin{aligned}
& \text { evenj-1 }=s j-1-U(d j-1) \\
& \text { oddj }-1=d j-1+P(\text { evenj }-1)
\end{aligned}
$$

These steps are illustrated in Figure 5 [5]

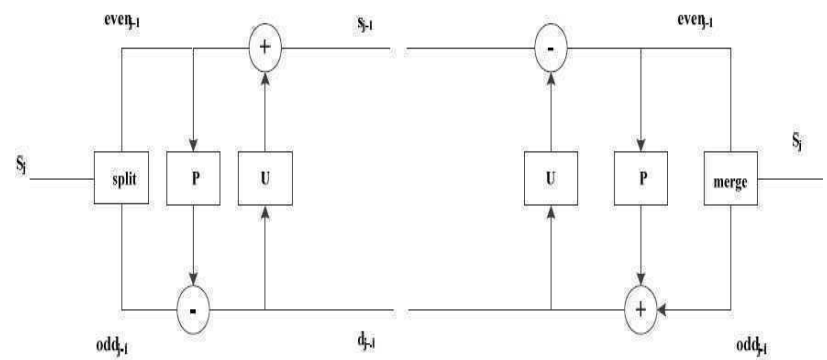

Figure 5: Direct and Inverse lifting step.

\section{A. Lifting scheme version of the Daubechies 4 Wavelet transforms}

The two steps of Lifting Scheme wavelet transform are update and predict. Here a new step is included named normalization, shown in figure 6 [1].

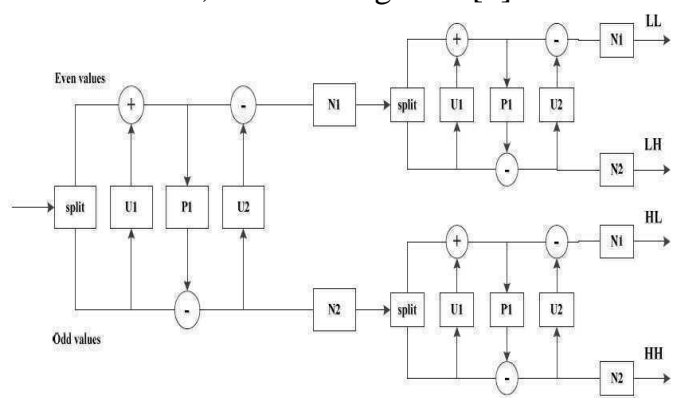

Figure 6: Two stages Daubechies $\mathrm{Db} 4$ forward lifting wavelet transform.

The input data in the split step is separated into even and odd elements. The even elements are kept in $\mathrm{S}_{0}$ to Shalf-1, the first half of an $\mathrm{N}$ element array section. The odd elements in $S_{\text {half }}$ to $\mathrm{S}_{\mathrm{n}-1}$, the second half of an $\mathrm{N}$ element array section. The equations below the expression Shalftn refers to odd element, $S_{n}$ refers to the even in the forward transform. The term LL refers to low frequency components and LH, HL, HH represents the high frequency components in the horizontal, vertical and diagonal directions respectively.

The forward step equations are

Update1 (U1):

For $\mathrm{n}=0$ to half- 1

$S[n]=S[n]+\sqrt{3} S[$ half $+n]$

Predict (P1):

$S[$ half $]=S[$ half $]-\frac{\sqrt{3}}{4} S[0]-\frac{\sqrt{3}-2}{4} S[$ half +1$]$

For $\mathrm{n}=1$ to half- 1

$S[$ half $+n]=S[$ half $+n]-\frac{\sqrt{3}}{4} S[n]-\frac{\sqrt{3}-2}{4} S[n+1]$

Update2 (U2):

For $\mathrm{n}=0$ to half- 2

$S[n]=S[n]-S[$ half $+n+1]$

$S[$ half -1$]=S[$ half -1$]-S[$ half $]$

Normalize (N)

For $\mathrm{n}=0$ to half -1

$S[n]=\frac{\sqrt{3}-1}{4} S[n]$

$S[n+$ half $]=\frac{\sqrt{3}+1}{4} S[n+$ half $]$

The addition and subtraction operations interchanged in the inverse transform, as the inverse transform is a mirror of the forward transform [6].

\section{SET PARTITIONING IN HIERARCHICAL TREES}

The image compression algorithms like Embedded Zero tree Wavelet (EZW), Set Partitioning in Hierarchical Trees (SPIHT). Image compression is of two types, Lossless and lossy compression. In Lossless compression, after performing decompression exactly the original image is obtained, in practice images with natural scenes error free compression is not possible. Coming to the lossy compression, there are methods in lossy compression such as EZW algorithm, SPIHT algorithm, WDR algorithm and ASWDR algorithm etc. These recent image compression algorithms have fewer errors per compression rate and the perceptual quality is also high. Compression of an image involves five stages as shown in the figure 7 and here is lossy compression only because of quantize step is not invertable, Quantizing is a quality reduction of the wavelet transform floating point values of 32-bit or 64-bit.

The important point here in wavelet based compression is the connection between Quantize, Encode step and the block diagram is shown in figure 7 [7]. 


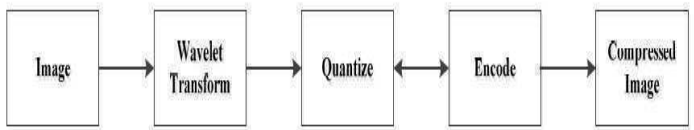

Figure 7: Block Diagram of a transform based coder.

The SPIHT algorithm achieved the higher PSNR values for a given compression ratios and high perceptual quality also the greater computational complexity is obtained by this algorithm. The drawback of SPIHT is that, it is difficult to perform on certain selected points or selected region of a compressed image where increase in resolution is required [7]. SPIHT is a powerful wavelet based image compression algorithm. It is a benchmark state of art algorithm for image compression.

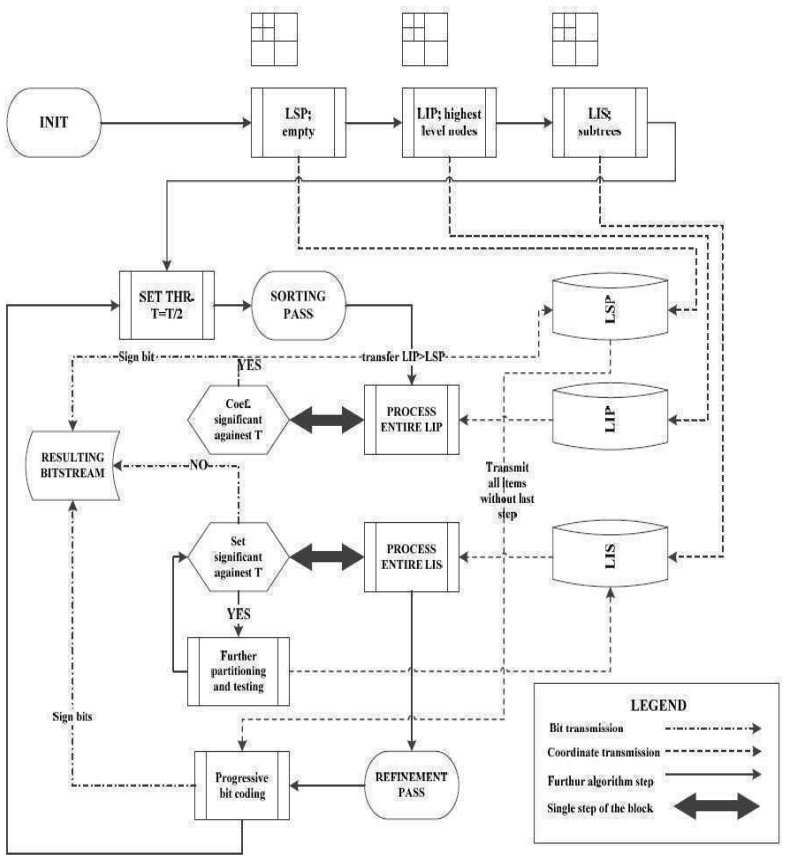

Figure 8: SPIHT algorithm scheme.

The operation of SPIHT goes on from testing the ordered wavelet coefficients for significance in a decreasing bit plane order, and just quantizing the significant coefficients. This algorithm achieves high coding efficiency because of group testing of the wavelet tree coefficients. This algorithm maintains the insignificant coefficients group in bigger subsets with the use of sequenced partitioning of trees. SPIHT algorithm does the grouping of wavelet coefficients and trees into sets depending on their significance information. Sorting and Refinement are the two stages used in SPIHT encoding algorithm. The significant wavelet transform coefficients are coded first and later the bits are transmitted as a result a more enhanced copy of the original image is obtained.

Following the initialization step, for each level of threshold, sorting pass and refinement pass are the next two stages. In the first stage i.e., sorting pass, SPIHT tries to group the coefficients accordingly depending on their magnitude. Quantization of the coefficients is improved in the refinement pass, where actual progressive coding transmission is done [4]. Relative to the threshold, sorting and refining is performed.

The threshold is set and without any interruption it is made smaller while operating throughout the algorithm. A form of bit-stream is obtained as output result. In the decomposition, SPIHT allows three lists of coordinates of coefficients. In the order, they are the List of Insignificant Pixels (LIP), the List of Significant Pixels (LSP), and the List of Insignificant Sets (LIS). At a certain threshold, a coefficient is considered significant if its magnitude is greater than or equal to threshold. The LIP, LIS, and LSP concept can be defined based on the idea of significance. SPIHT algorithm in detail is shown and explained in the figure 8 [8].

\section{IMAGE FUSION}

The process of getting important information from two or more images and combining it into a single image is called Image Fusion. The resultant image contains more information compared to each of the input images. Remote sensing applications give access to large amount of space borne sensors which provides a motivation for various image fusion algorithms. High spatial and spectral resolutions are needed in a single image in today's situation. Image fusion is the solution for it, as this technique allows the integration of several information sources and hence the output fused image contains attractive spatial and spectral resolution characteristics. In case of multispectral data, while merging the spectral information can be distorted by the standard image fusion techniques. High Pass Filtering is the basic method for image fusion. The other techniques available are IHS transform based image fusion, PCA based image fusion, and Wavelet transforms image fusion, Pair-wise spatial frequency matching. These techniques are based on DWT, Uniform rational filter bank, and laplacian pyramid.

DWT is very useful tool for fusion. For images to perform image fusion, they must be registered before fusion. The major applications of image fusion are classification of image, Aerial and Satellite imaging, Medical Imaging, Robot Vision, Concealed weapon detection, Multi focus-image fusion, Digital Camera Application, Battle field Monitoring etc.

Wavelet transform fusion is defined by considering the wavelet transforms $\varphi$ of the two or more registered images $g_{1}(x, y), g_{2}(x, y), g_{3}(x, y)$ together with the fusion rule. Inverse wavelet transform $\varphi^{-1}$ is computed, and the fused image is reconstructed.

$g(x, y)=\varphi-1(\sigma(\varphi(g 1(x, y)), \varphi(g 2(x, y)), \varphi(g 3(x, y)))$

Directional information is obtained from wavelet transform, i.e. low-low (LL), high-low (HL), low-high $(\mathrm{LH})$, and high-high $(\mathrm{HH})$ bands, which contains unique information and the process is shown in figure 9 [9].

FFT based technique has been used to register the three low resolution images as proposed in introduction. Each low resolution image is down sampled using two level biorthogonal filter based discrete wavelet transform and 
later these are fused. Here approximate and detail coefficients are fused.

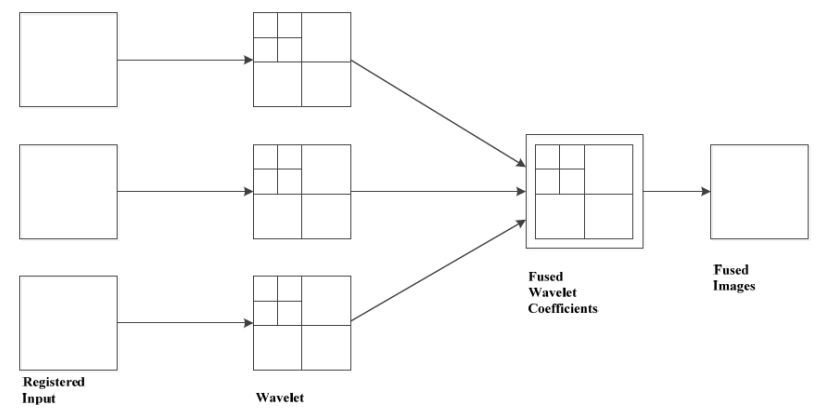

Figure 9: DWT based fused image.

The fusion of decomposed images is done by using fusion rule i.e. Maximum Frequency Fusion: "fusion by averaging for each band of decomposition and for each channel the wavelets coefficients of the three images are averaged". That is

approximate and detail coefficients are fused separately

$$
\begin{aligned}
& M_{j}{ }^{c} I=\left(M_{j}{ }^{c} I_{1}+M_{j}{ }^{c} I_{2}+M_{j}{ }^{c} I_{3}\right) / 3 \\
& N_{j}{ }^{\mathrm{o}} I=\left(N_{j}{ }^{\mathrm{o}} I_{1}+{N_{j}}^{\mathrm{o}} I_{2}+{N_{j}}^{\mathrm{o}} I_{3}\right) / 3
\end{aligned}
$$

The fused image is obtained by using Inverse wavelet transform. The resulting image is a de-noised image, the blur present in the image is removed using Iterative Blind Deconvolution. The high resolution image is obtained from wavelet based interpolation.

\section{DECONVOLUTION METHODS}

The blur present in the image is removed using Deconvolution methods.

\section{A .Blind Deconvolution}

The standard de-convolution problem in the frequency domain is given as

$\mathrm{G}(\mathrm{Kx}, \mathrm{Ky})=\mathrm{F}(\mathrm{Kx}, \mathrm{Ky}) \mathrm{H}(\mathrm{Kx}, \mathrm{Ky})+\mathrm{N}(\mathrm{Kx}, \mathrm{Ky})$

Here we need to estimate the input spectrum $\mathrm{F}(\mathrm{Kx}, \mathrm{Ky})$. The noise spectrum $\mathrm{N}(\mathrm{Kx}, \mathrm{Ky})$ is unknown. Also if the systems Optical Transfer Function (OTF) H (Kx,Ky) is also an unknown then de-convolution problem occurs. By looking at the equation this blind de convolution problem seems impossible as measured output system $\mathrm{G}(\mathrm{Kx}, \mathrm{Ky})$ is equal to product of two unknown quantities plus a random noise process.

The solution for this problem can be found by iterative procedures carried out in frequency domain. The basic constraints of the solutions are that they have finite support, i.e. the sought input distribution is known to be confined to certain spatial region and is zero outside this region. The Blind de-convolution method not only estimates or restores the original input distortion but also the PSF responsible for the degradation.

\section{B. Iterative Blind Deconvolution}

Iterative Blind De-convolution technique is a kind of de-blurring filter used to remove blur in the image. The Blind de-convolution of two convolved functions is shown by a simple iterative method. Mathematically, the convolution $\mathrm{c}(\mathrm{x})$ of two functions, $\mathrm{f}(\mathrm{x})$ and $\mathrm{g}(\mathrm{x})$ is given by integral equation

$$
c(x)=\int_{- \text {inf }}^{+ \text {inf }} f(x 1) g(x-x 1) d x 1
$$

The Fourier transform representation of the above equation is

$$
\mathrm{C}(\mathrm{u})=\mathrm{F}(\mathrm{u}) \mathrm{G}(\mathrm{u})
$$

The basic de-convolution method consists of the following steps. First, a non negative valued initial estimation $f_{o}(x)$ is input into the iterative scheme. This function is Fourier transformed to yield $F^{\wedge}(u)$, which is then inverted to form an inverse filter and multiplied by $C(u)$ to form a first estimate of the second functions spectrum Go $(u)$. This estimated Fourier spectrum is inverse transformed to give $\mathrm{g}_{\mathrm{o}}(x)$. The image-domain constraint of non-negativity is now imposed by putting to zero all points of the function go $(x)$ that have a negative value. A positive constrained estimate $\mathrm{g}_{0} \wedge(x)$ is consequently formed that is Fourier transformed to give the spectrum $\mathrm{Go}^{\wedge}(u)$. This is inverted to form another inverse filter and multiplied by $\mathrm{C}(\mathrm{u})$ to give the next spectrum estimate $\mathrm{F}_{1}(u)$. A single iterative loop is completed by inverse Fourier transform $\mathrm{F}_{1}(u)$ to give $\mathrm{f}_{1}(u)$ and by constraining this function to be nonnegative, yielding the next function estimate $\mathrm{f}_{1} \wedge(x)$. The iterative loop is repeated until two positive functions with the required convolution.

Blind de-convolution is a technique that allows the reconstruction of image from a single or more blurred image in the presence of a poorly determined or unknown PSF. Blind de-convolution can be performed iteratively where each iteration improves the estimation of the PSF and the scene IBD starts with an initial estimate of the restored image, an initial estimate of the PSF restoring the image is by making an initial estimate of what the PSF and image are. One of the constraints that we apply to the image is that of finite support. Finite support basically says that the image does not exist beyond a certain boundary. The first set of Fourier constraints involve estimating the PSF using the FFT of the degraded image and FFT of the estimated PSF

$$
H k(u, v)=\frac{G(u, v) \operatorname{conj}(F 1(u, v))}{|F 1(u, v)| \wedge 2+\operatorname{alpha}|F(u, v)| \wedge 2}
$$


By applying IFFT to $H k(u, v)$, we obtain the PSF. The true image is restored by deconvolution of the PSF with the degraded image. Hence the second set of constraints Involve

$$
F k(u, v)=\frac{G(u, v) \operatorname{conj}(H 1(u, v))}{|H 1(u, v)| \wedge 2+\operatorname{alpha}|H(u, v)| \wedge 2}
$$

The blur constraints that are applied are from the assumptions that we know or have some knowledge of the size of the PSF [10].

\section{Image Quality Measurement}

The image quality is measured from the parameters Mean Square Error (MSE), Structural Similarity Index Metric (SSIM) and Mean SSIM (MSSIM)

\section{(i)Mean Square Error (MSE)}

The two metrics are used to measure the performance index of image compression are Objective Fidelity criteria and Subjective Fidelity criteria. MSE is a measure belongs to Objective Fidelity criteria. The energy lost in the lossy compression of the original image $f$ is estimated using this. If we consider two images affected by same type of degradation, the image with low MSE is closer to the original image. The Mean Square Error between the original image $f(m, n)$ and the reconstructed image $g(m, n)$, which contains $\mathrm{M} \times \mathrm{N}$ pixels is

$$
\begin{aligned}
& \left.M S E=\frac{1}{M * N} \sum_{m=0}^{M-1} \sum_{n=0}^{N-1}(f(m, n))-g(m, n)\right) 2 \\
& P S N R=10 * \log 10\left[\frac{(2 b-1) 2}{M S E}\right]
\end{aligned}
$$

For an 8-bit image, $\mathrm{b}=8$ which gives the PSNR value as

$$
P S N R=10 * \log 10\left[\frac{2552}{M S E}\right]
$$

\section{(ii)Structural Similarity Index Metric (SSIM)}

SSIM, Structural Similarity Index Metric is an objective video quality assessment metric used in predicting the video quality. The structural similarity among two frames is calculated by using SSIM. An alternative in evaluation of perceptual quality is SSIM.

It takes the quality degradations in the frames as perceived changes in the variation of structural information among two frames

$$
\operatorname{SSIM}(\mathrm{x}, \mathrm{y})=\frac{\left(2 \mu_{x} \mu_{y}+C_{1}^{\prime}\right)\left(2 \sigma_{x y}+C_{2}^{\prime}\right)}{\left(\mu_{x}^{2}+\mu_{y}^{2}+C_{1}^{\prime}\right)\left(\sigma_{x}^{2}+\sigma_{y}^{2}+C_{2}^{\prime}\right)}
$$

where $\mu_{\mathrm{x}}, \mu_{\mathrm{y}}$ are the average of $\mathrm{x}$ and $\mathrm{y}$ respectively, $\sigma_{\mathrm{x}}$, $\sigma_{\mathrm{y}}$ are the variance of $\mathrm{x}$ and $\mathrm{y}$ respectively, $\sigma_{\mathrm{xy}}$ is the covariance of $\mathrm{x}$ and $\mathrm{y} . \mathrm{c}_{1}, \mathrm{c}_{2}$ are the constants used in order to evaluate any instabilities in the structural similarity comparison.

The Mean SSIM index to evaluate the overall image quality

$$
\operatorname{MSSIM}(X, Y)=\frac{1}{M} \sum_{j=1}^{M} \operatorname{SSIM}(x j, y j)
$$

where $\mathrm{M}$ is number of local windows in the image [11].

\section{IMPLEMENTATION}

The growing requirement for high resolution images resulted in the need for super resolution image reconstruction. Our goal in creating a super resolution image is to consider different types of same scene image of low resolution as inputs and combine them to generate a high resolution image through a series of steps. The implementation consists of taking either a source image for developing low resolution images like blurred, noisy and rotated versions of it as shown in Figure 10. The noisy image is generated by mixing the signal with a statistical random noise in particular an Additive White Gaussian noise. Such a noise component has a zero mean and constant power spectral density. The value at each time is uncorrelated to the value at any other time instant in Gaussian noise components. The blurred version is generated by convolution of the image with a window of chosen value. The convolution by a windowed function decreases the amplitude at some points and increases the amplitude at other points thereby generated a blurred image. Finally the rotated version is generated by inbuilt Matlab function viz.imrotate( ). By providing the arguments label of the image, angle through which it should be rotated, size of the returned image along with method of interpolation. Bicubic interpolation is chosen that provides pixel outside the image range to rectify for pixels lost due to rotation. The 'crop' option returns the image of same size as that of source image.

The next step in the process is image registration which influence only on rotated, translated, scaled and shear images and passes all other images without any action. By comparing with reference images the rotated image input(s) are oriented back to its original form. It is the process of overlaying two or more images of the same scene taken at different times, from different viewpoints and or by different sensors. To a base image taken as reference the other images are compared. Spatial transformation helps in orienting the input images in the same direction as base or reference image. Using the translational, rotational, scaled and sheared estimations of translational, rotational, scaled and sheared images constructs the base image from spatial transformations. The output of this step is an image oriented as input image along with noisy and blurred versions that are passed through.

For color images, R G and B components are separated from each image and Lifting Wavelet Transform (LWT), is applied to each component using Daubechies 4 
coefficients. Daubechies wavelets are a family of orthogonal wavelets defining a discrete wavelet transform and characterized by a maximal number of vanishing moments for some given support. With each wavelet type of this class, there is a scaling function (called the father wavelet) which generates an orthogonal multi resolution analysis. A wavelet transform for an image implies that it is sampled both in frequency and time to form wavelets. A two level decomposition is applied such that after decomposing an image using first level decomposition it retains maximum information in LL band. The 'LL' band is decomposed for second time so that available information is concentrated in a very small band 'LLLL'. Each output image is compressed by a powerful wavelet based image compression method known as SPIHT. The output from the two level transformations is encoded and then decoded by SPIHT method. The output differs from the input in a way that is compressed without any loss of data, with high quality and without any error.

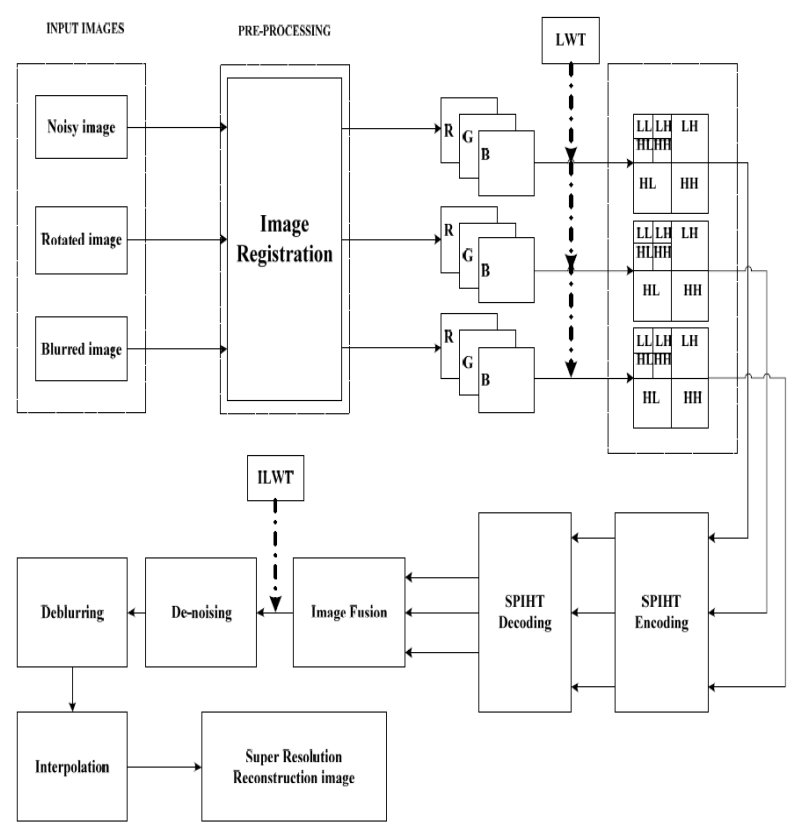

Figure 10: Block diagram for Super resolution image reconstructed

The three outputs are fused together to form a single image by a process of image fusion. The fusing technique used here is averaging, a sub class of spatial domain fusion along with transform domain.

The noise component of the image is removed by using a Dual tree Discrete Wavelet Transform abbreviated as Dual Tree DWT. The transform estimates the original image adaptively from the noise image by taking mean square error as optimisation criteria. The blurred part of the image is removed by using iterative blind deconvolution or blind deconvolution. Blind deconvolution is a process where in a target image is obtained from a single or set of blurred images by deducing its point spread function (PSF).
The final step in construction of a super resolution reconstruction image is interpolation of samples. Interpolation appends as many samples as needed between two input samples to generate band limited signal by applying a low pass filter. It is just the opposite of sampling process. The data points in the image increases by appending more samples between two input samples. The interpolation method used here is bicubic interpolation since images resampled with bicubic interpolation are smoother than bilinear interpolation. So by integrating three low resolution versions of same scene image over an algorithmic procedure, the resolution of the image is enhanced to obtain a super resolution reconstructed image.

\section{RESULTS}

Image Quality Measurement values for proposed method are listed in table 1 .

TABLE I

SSIM VALUE FOR PROPOSED CASE FOR LENA IMAGE \& HD IMAGE

\begin{tabular}{|c|c|c|c|c|c|c|c|}
\hline \multirow{2}{*}{$\begin{array}{l}\text { Image } \\
\text { Quality } \\
\text { Metric }\end{array}$} & \multicolumn{3}{|c|}{ Input Images } & \multicolumn{4}{|c|}{$\begin{array}{c}\text { Super Resolution } \\
\text { Reconstruction Image }\end{array}$} \\
\hline & $\begin{array}{l}\text { nois } \\
\mathrm{e}\end{array}$ & $\begin{array}{l}\text { blurre } \\
\text { d }\end{array}$ & $\begin{array}{l}\text { rotat } \\
\text { ed }\end{array}$ & $\begin{array}{l}\text { after } \\
\text { Ima } \\
\text { ge } \\
\text { Fusi } \\
\text { on }\end{array}$ & $\begin{array}{l}\text { after } \\
\text { deno } \\
\text { ising }\end{array}$ & $\begin{array}{l}\text { after } \\
\text { BD }\end{array}$ & $\begin{array}{l}\text { after } \\
\text { IBD }\end{array}$ \\
\hline $\begin{array}{l}\text { SSIM for } \\
\text { Lena Image }\end{array}$ & $\begin{array}{c}0.57 \\
8\end{array}$ & 0.686 & $\begin{array}{c}0.22 \\
9\end{array}$ & $\begin{array}{c}0.80 \\
44\end{array}$ & $\begin{array}{c}0.81 \\
38\end{array}$ & $\begin{array}{c}0.75 \\
34\end{array}$ & $\begin{array}{c}0.77 \\
44\end{array}$ \\
\hline $\begin{array}{l}\text { SSIM for HD } \\
\text { Image }\end{array}$ & $\begin{array}{c}0.53 \\
87\end{array}$ & $\begin{array}{c}0.756 \\
5\end{array}$ & $\begin{array}{c}0.28 \\
68\end{array}$ & $\begin{array}{c}0.81 \\
71\end{array}$ & 0.83 & $\begin{array}{c}0.76 \\
58\end{array}$ & $\begin{array}{c}0.82 \\
45\end{array}$ \\
\hline
\end{tabular}

From the below represents The low resolution source image from which versions of it were developed is shown in figures 10, Fig (a), Fig (b) represents the noisy image derived from source image by adding an additive white Gaussian noise with variance 0.05 . Fig (c) is the rotated version of source image by an angle of 10 degrees. It was noted that rotation was checked for different angles and results coalesce. Fig (d) is the blurred version of source image developed by convolution with an impulse

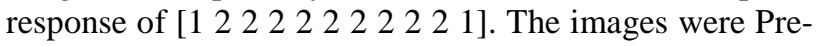
processed using image registration with arguments such that it applies to only rotational, translational and scaled images. By overlaying with reference images provided, the orientation of rotated image aligns similar to that of reference image. Fig (e) represents the super resolution reconstructed image after average fusing the three images obtained after applying LWT, two level decomposition, SPIHT encodes and SPIHT decodes in order. Fig (f) represents the image where noise is suppressed, obtained by applying dual tree DWT and interpolation. Fig (g) and Fig (f) represents the super resolution reconstructed images after reducing blur by blind deconvolution and iterative blind deconvolution respectively and are interpolated to twice the samples. 


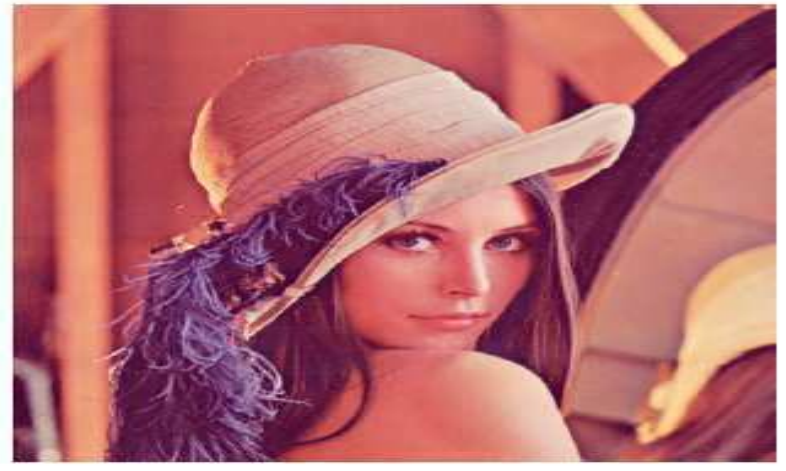

(a)

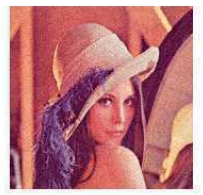

(b)

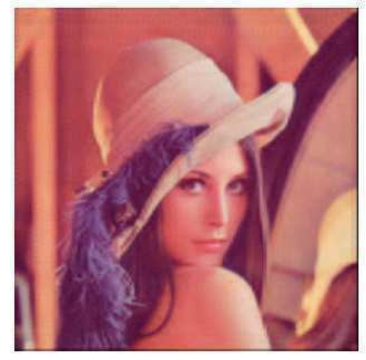

(e)

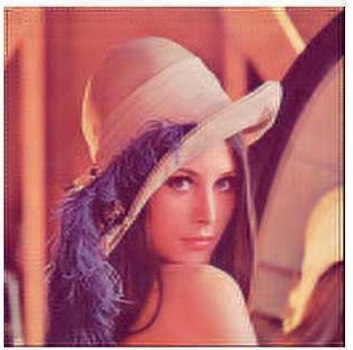

(g)

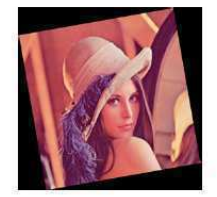

(c)

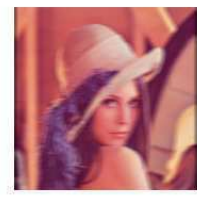

(d)

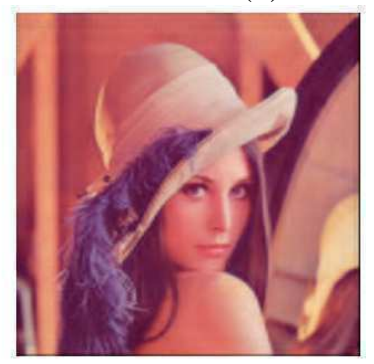

(f)

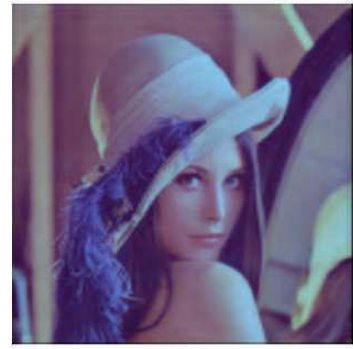

(h)

Figure 11: (a) Source image(512x512), (b) Noisy image(256x256), (c) Rotated image(256x256), (d) Blurred image(256x256), (e) SRR image after Image fusion(512x512), (f) SRR image after Denoising(512x512), (g) SRR image after Blind deconvolution(512x512),(h) SRR image after Iterative blind deconvolution $(512 \times 512)$.

\section{CONCLUSIONS}

It can be concluded that output is a high resolution version of input image with higher perceptual quality judging from the value of structural similarity (SSIM) with reference to input image shown in Table 1 . We obtained the results for different varieties of input images i.e. noisy, blurred, translated and rotated images. Image

fusion is applied and improved SSIM values are noted. However some blur and noise component is observed in the image. Denoising is performed by Dual tree DWT based algorithm and enhance SSIM values are noted. Blur component is suppressed by Blind Deconvolution (BD) and Iterative Blind Deconvolution (IBD). Also it can be concluded that debluring using IBD yields better SSIM values compared to $\mathrm{BD}$ so the former one is more advantageous than later but this comes at a higher computational cost. The results from proposed models concludes that a high resolution image can be achieved by combining two or more low resolution images of same scene. The key to the success of the algorithm is having an accurate observation model. This includes the image registration parameters and the system PSF.

\section{REFERENCES}

[1]. C. N. R. Kumar, "A Novel and Efficient Lifting Scheme based Super Resolution Reconstruction for Early Detection of Cancer in Low Resolution Mammogram Images," International Journal of Biometrics and Bioinformatics (IJBB), vol. 5, no. 2, p. 53, 2011.

[2]. B. S. Reddy and B. N. Chatterji, "An FFT-based technique for translation, rotation, and scaleinvariant image registration," Image Processing, IEEE Transactions on, vol. 5, no. 8, pp. 1266-1271, 1996.

[3]http://www.bearcave.com/misl/misl_tech/wavelets/lifting/ind ex.html

[4]. Sapan Naik, Viral Borisagar," A novel Super Resolution Algorithm using Interpolation and LWT based denoising method ", IJIP journal, vol. 6, issue 4, 2012.

[5]. A. Jensen and A. la Cour-Harbo, Ripples in mathematics: the discrete wavelet transform.Springer, 2001.

[6]http://www.bearcave.com/misl/misl_tech/wavelets/daubechie s/index.html

[7] J. S. Walker and T. Q. Nguyen, "Wavelet-based image compression," The Transform and Data Compression Handbook Ed. KR Rao et al. Boca Raton, CRC Press LLC, 2001.

[8] J. Malỳ and P. Rajmic, "DWT-SPIHT IMAGE CODEC IMPLEMENTATION," Department of telecommunications, Brno University of Technology, Brno, Czech Republic.

[9] Liyakathunisa, C.N. Ravi Kumar "Advances in Super Resolution Reconstruction of Noisy Blurred and Aliased Low Resolution Image", International Journal of Computational Intelligence Research ISSN 0973-1873 Volume 6, Number 2 (2010), pp. 215-236.

[10] G. R. Ayers and J. C. Dainty, "Iterative blind deconvolution method and its applications," Optics letters, vol. 13, no. 7, pp. 547-549, 1988.

[11] Z. Wang, A. C. Bovik, H. R. Sheikh, and E. P. Simoncelli, "Image quality assessment: From error visibility to structural similarity," Image Processing, IEEE Transactions on, vol. 13, no. 4, pp. 600-612, 2004. 